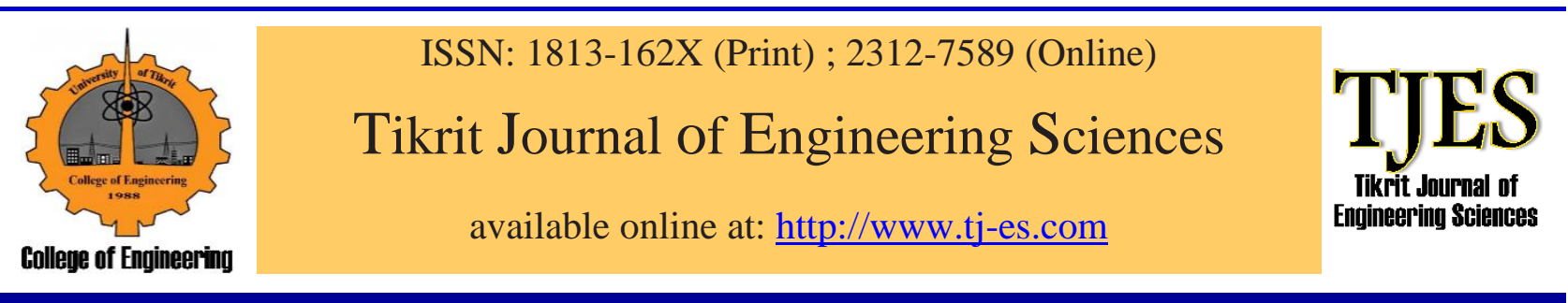

Razooqi RN, Akbar AA, Hamada ML. Influence of the Yttria and Graphite Particles on the same of Al 2024 Alloy Properties. Tikrit Journal of Engineering Sciences 2019; 26(4):1-7.

\section{Raed N. Razooqi ${ }^{1}$ \\ Ahmed A. Akbar ${ }^{2}$ Mohanad L. Hamada*1}

${ }^{1}$ Mechanical Eng. Dept./ College of Eng./ Tikrit University

${ }^{2}$ Dept. of Production Eng. And Metallurgy/ University of Technology

\title{
Influence of the Yttria and Graphite Particles on the same of Al 2024 Alloy Properties
}

\section{A B S T R A C T}

Powders have been mixed as (Al with $99 \%$ purity $\leq 53 \mu \mathrm{m}$ particle size, $\mathrm{Cu}$ with $99.5 \%$ purity $\leq 53 \mu \mathrm{m}$ particle size, $\mathrm{Mg}$ with $99.5 \%$ purity $\leq 53 \mu \mathrm{m}$ particle size, $\mathrm{Y} 2 \mathrm{O} 3$ with $99.9 \%$ purity $\leq 53 \mu \mathrm{m}$ particle size, Gr with $99.9 \%$ purity $\leq 53 \mu \mathrm{m}$ particle size). one set of composite samples was prepared by addition $(3 \mathrm{wt} \% \mathrm{Gr})$ to metal powders shown above. For the each sample of composite varying (Y2O3) weight percentage as: (Al 2024-3wt\%Gr-XY2O3) was added $\mathrm{x}=(0,2,4,6,8)(\mathrm{wt} \%)$ the other set of composite was in opposite. Well mixed samples was pressed uniaxial with (700Mpa) and $(30 \mathrm{sec})$. the compacted samples were sintered at (6000C) for $(2 \mathrm{hr})$. Results have shown an increase in bulk density by (5.3\%) for (Al 2024-3wt\% Gr-XY2O3) with the increase of reinforcement until (8wt\%) of (Y2O3), while bulk density have been reduced for ( $\mathrm{Al} 2024-3 \mathrm{wt} \% \mathrm{Y} 2 \mathrm{O} 3-\mathrm{XGr})$ with $(5 \%)$ with the increase of $(\mathrm{Gr})$ weight content in the composite. An increase in real porosity was noticed with the increase of (Y2O3) with (8wt\%) (11.9\%) for (Al 2024-3wt\%Gr-XY2O3) but this was less in comparison with (Al 2024-3wt\% Y2O3-XGr) were porosity increase with the increase of $(\mathrm{Grwt} \%)$ up to reduced $(18.5 \%)$ at $(8 \mathrm{wt} \% \mathrm{Gr})$.Mean value was of micro hardness decreased gradually with the increase of (Grwt\%) to reach $(19.4 \%)$ reduction at $(8 \mathrm{wt} \% \mathrm{Gr})$ in opposite the micro hardness mean value increased by $(20 \%)$ as $(\mathrm{Y} 2 \mathrm{O} 3 \mathrm{wt} \%)$ increased to $(8 \mathrm{wt} \%)$.Compression strength have was increased by $(51 \%)$ as (Y2O3) content increased from (2-8)(wt\%) for (Al 2024-3wt\%GrXY2O3) composite, while it was decreased by (48\%) with the addition of (8wt\%) graphite to ( $\mathrm{Al}$ 2024-3wt\%Y2O3-XGr).The content of (Gr) had higher effect on wear rate than (Y2O3) where the wear rate decreased by $(59.7 \%)$ and $(41 \%)$ for composite samples respectively, within the limits of addition in the current study.

(C) 2019 TJES, College of Engineering, Tikrit University

DOI: http://doi.org/10.25130/tjes.26.4.01

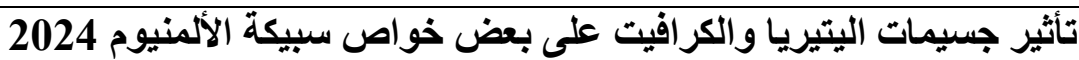

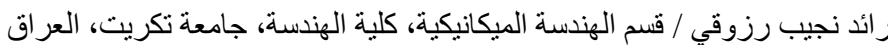

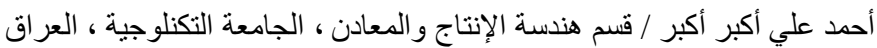

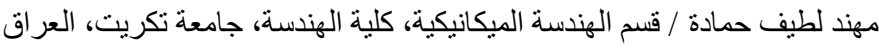
الخلاصة

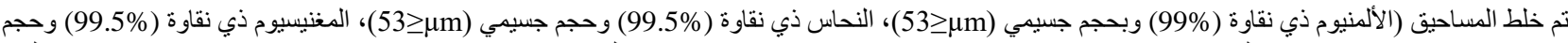

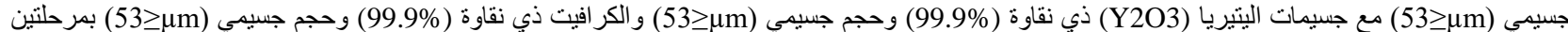

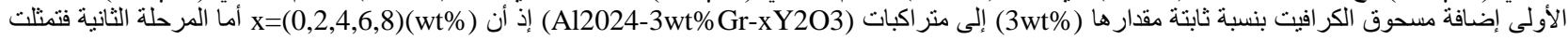

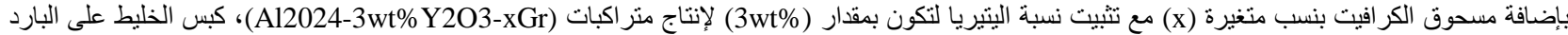

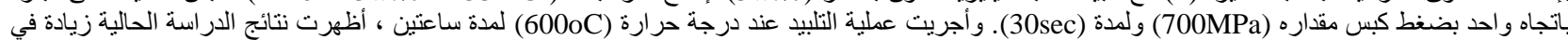

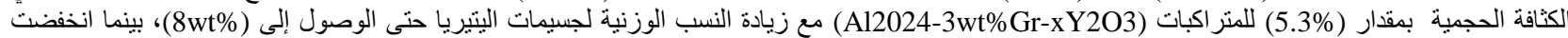

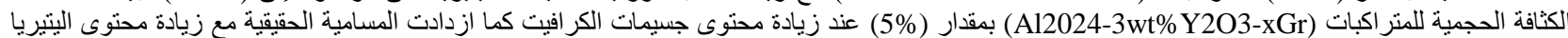

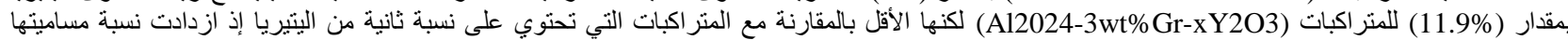

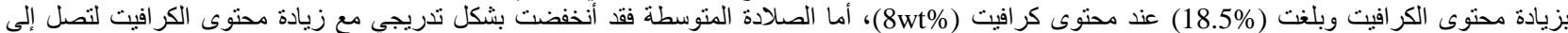

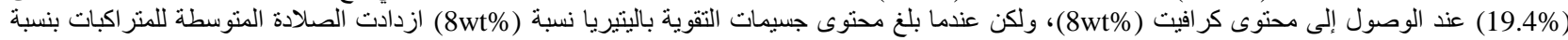

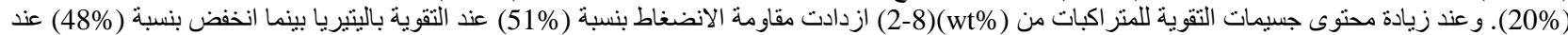

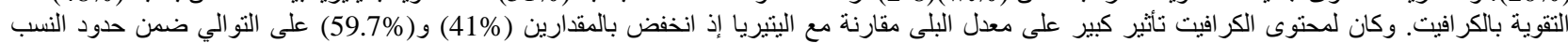
المضافة قيد الدر اسة. الكلمات الدالة: اليتيريا ، الكر افيت ، سبيكة الألمنيوم 2024

\footnotetext{
* Corresponding Author: E-mail: muhanad.lateef@yahoo.com
} 
بتقانة مينالورجيا المسـاحيق. استخدم الباحثنان مسـحوق الألمنيوم التجاري ذو نقاوة (99.8\%) وبحجم جسيمات (40-325Mesh)

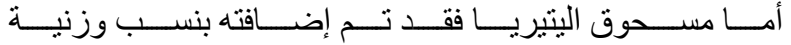

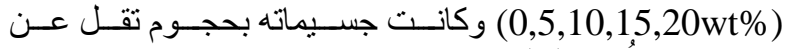

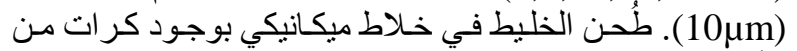

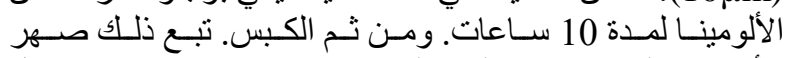

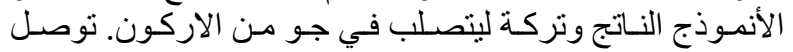

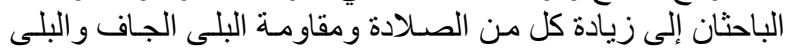

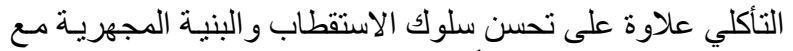

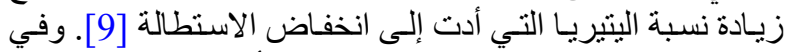
عام 2009 درس الباحث

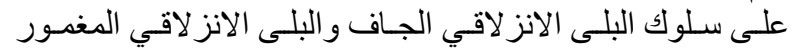

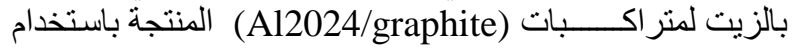

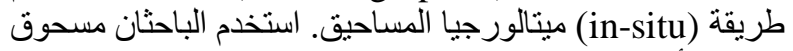

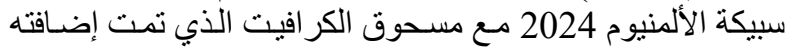

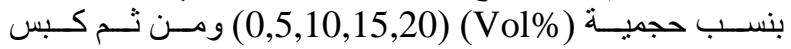
المسـوق على البـارد بضـغط قدرة (650Mpa). لبدت العينـات

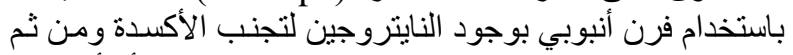
تم تلبيد العينات التي تحتوي على نسبة (15Vol\%) أو أقل من أنس

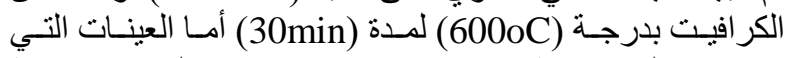

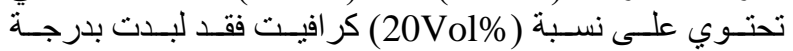
(610oC)

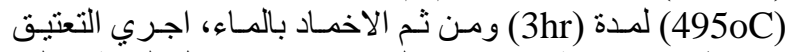

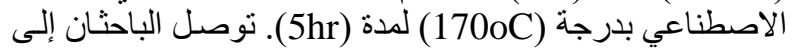

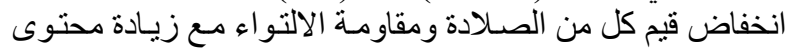

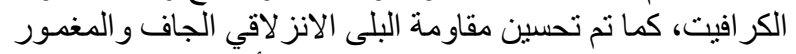

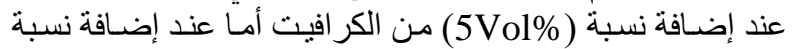
(10Vol\%) أو أكثر ازداد معدل البلى[10].

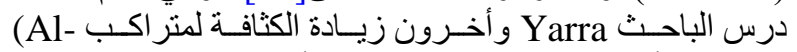

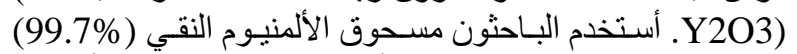

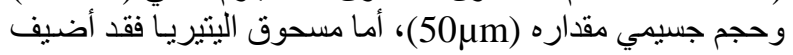

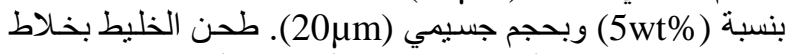

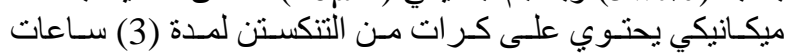

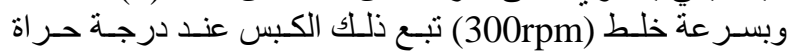
الغرفة بتمريرنين في ممر زاوي منساو

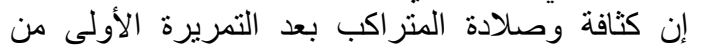

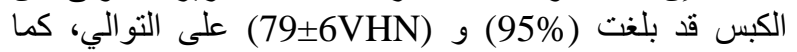

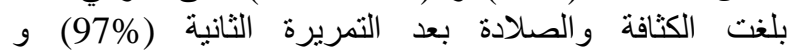
على التوالي أيضا وحصل الباحثون على (83.2_4.7 VHN) بنية مجهرية متجانسة ومقاومة ميكانيكية عالية للمتر اكب البولئ [113]. وفي عام 2013 درس الباحث Ravindran السلوكئك

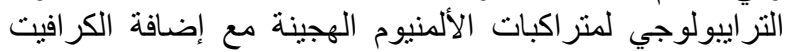

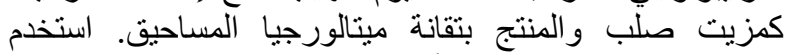
الباحثون مسحوق سبيكة الألمنيوم 2024 مع مسحوق مستيثة كاربيد

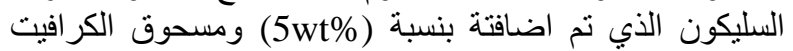
بنسبة (0,5,10wt\%). خلطت المساحيق باستخدام ماكنة خلط كوكبية مع وجود الكرات ومن ثم كبس الخليط باستخدام جهاز

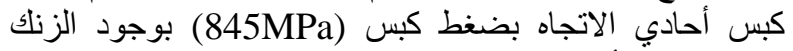

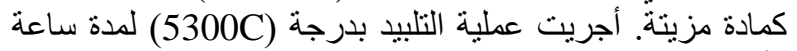

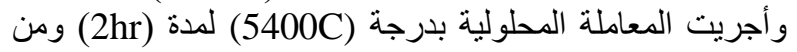

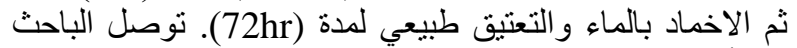

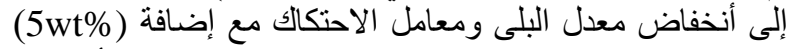

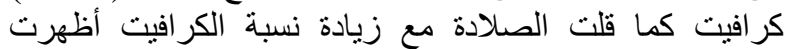

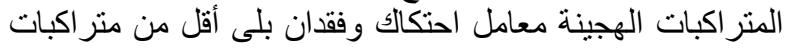

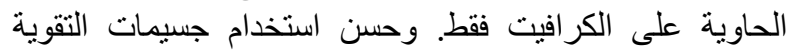
وجسيمات الكرافيت في المنر اكبات الهجينة من الخواصن (Sic) 


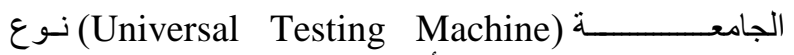
(180KN) ذات منشـأ صـبني وبسـعة كبـ (WDW-200E)

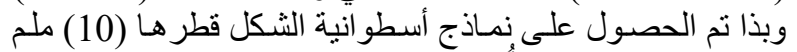

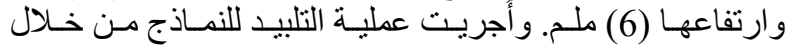

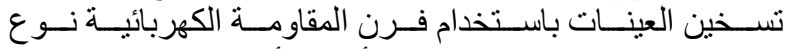
إنكليزي المنشأ تصل أقصى درجة حر ارة لـه له

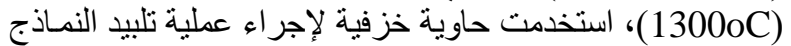

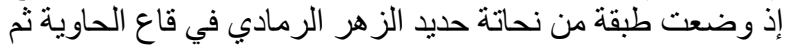

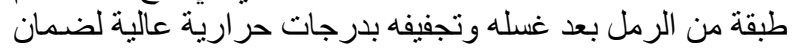

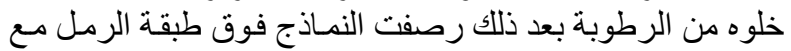

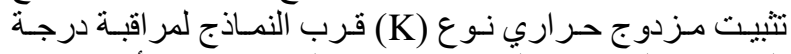

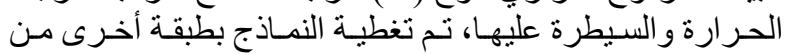

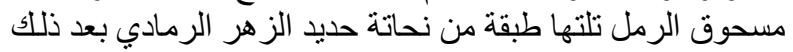

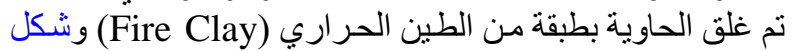

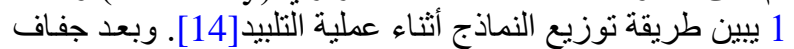

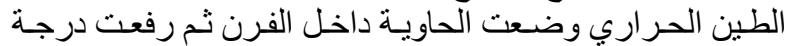

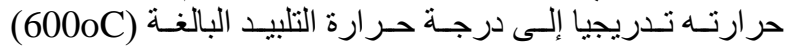

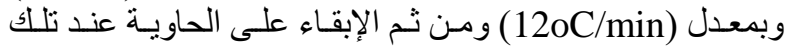

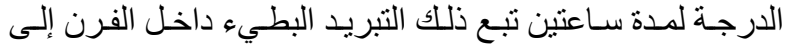
درجة حر ارة الغرفة

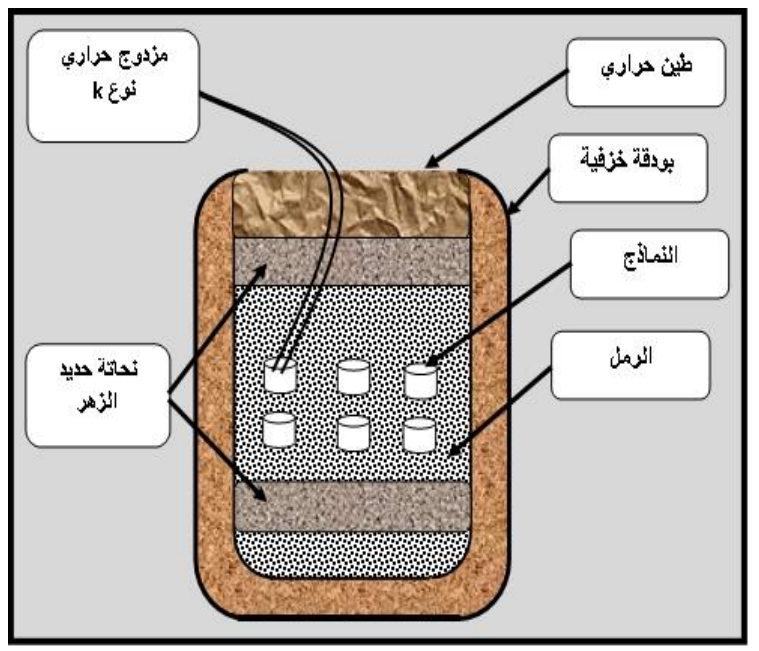

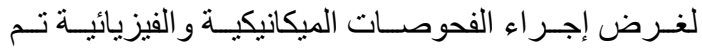

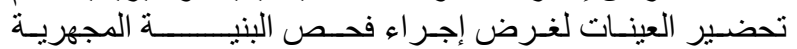

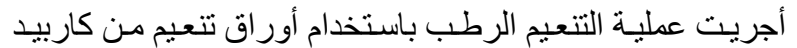
السليكون بعدة مر احل هي (500,1000,1500,2500) ثم صقلت كالت

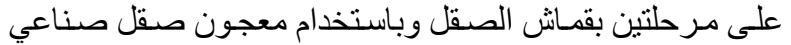

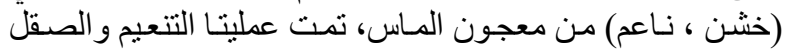

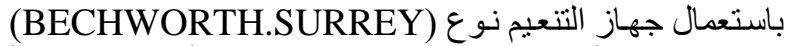
إنكليزي المنشـأ وجهاز الصـقل (ECOMET) أمريكيكي المنشـأ

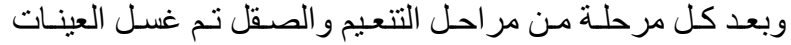

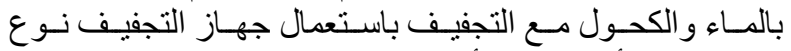

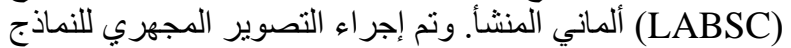

باستخدام المجهر الضوئي نوع (OLYMPUS) ياباني المنشأ. تم تحديد الأطوار باستخدام جهاز فحص حيود الأشـعة السينية

شكل 1: الحاوية الخزفية ومحتوياتها أثناء عملية التلبيد [14].

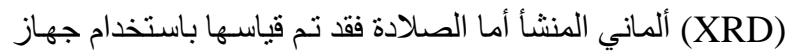

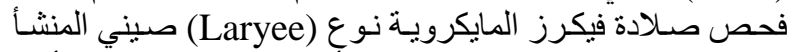

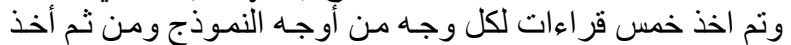

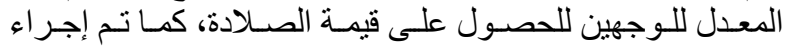

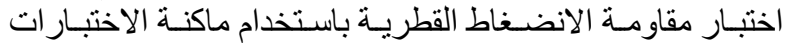
الجامعـة نـوع (WDW-200E) و أجريـت اختبـار مقاومسة البلى الانى
الترايبولوجية لنظام الانزلاق للمواد المنتجة باستخدام تقانة ميتالورجيا المساحيق [12] ـ

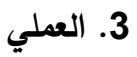

يوضـح جدول 1 أنواع المسـاحيق المستخدمة في تصنيع

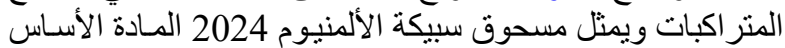

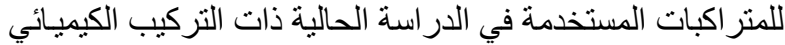

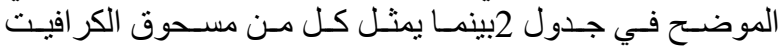

ومسحوق اليتيريا مو اد التقوية.

جلول 1 أنواع المساحيق المستخدمة في تصنيع المتر اكبات

\begin{tabular}{|c|c|c|c|c|}
\hline المنشأ & 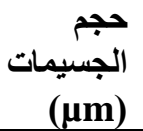 & $\begin{array}{r}\text { (النقاوة } \\
\text { (\%) }\end{array}$ & المسحوق & ت \\
\hline شركة من شنH & $53 \geq$ & 99 & $\begin{array}{l}\text { الألمنيوم } \\
\text { (AL) }\end{array}$ & 1 \\
\hline شندي من شن شنH & $53 \geq$ & 99.5 & $\begin{array}{r}\text { النحاس } \\
\text { (Cu) }\end{array}$ & 2 \\
\hline $\begin{array}{r}\text { شركة } 1 \text { شرني } \\
\text { RIERDEL- } \\
\text { DEHAEN } \\
\text { AG }\end{array}$ & $53 \geq$ & 99.5 & المغنيسبوم & 3 \\
\hline 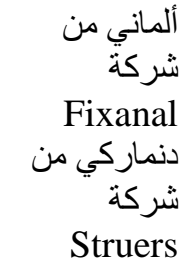 & $53 \geq$ & 99.9 & (Y2O3) & 4 \\
\hline
\end{tabular}

إذ يتكون الجانب العملي من مرحلتين الأولى إضافة

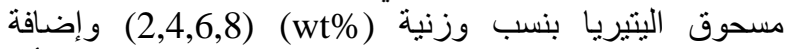
مسحوق الكرافيت بنسبة وزنيه ثابتة مقدارها (3wt\%) أما

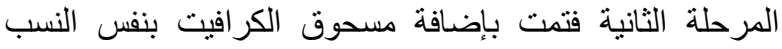

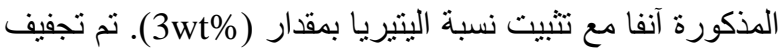

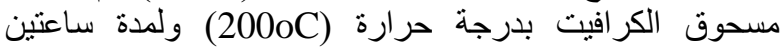

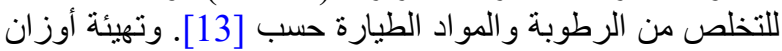
خلطة كل نوع من أنواع المتراكبات بإتباع النسب الوالديب الوزنية بين سبيكة الألمنيوم 2024 وكل من اليتيريا و الكر افيت.

جدول التركيب الكيميائي لسبيكة الألمنيوم 2024.

\begin{tabular}{rccc}
\hline Metal & AL & $\mathrm{Cu}$ & $\mathrm{Mg}$ \\
\hline$($ wt \%) & Bal. & 4.5 & 1.5 \\
\hline
\end{tabular}

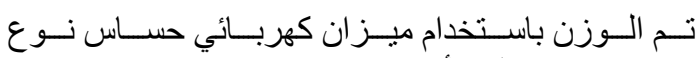
سويسري المنشأ وبدقة قياس (Precisa) ثم وضعت أوزان العناصر المكونة لكل متر اكب في داخل الحند الحاوية

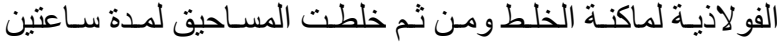

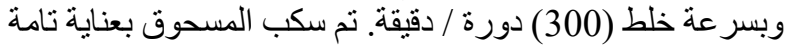

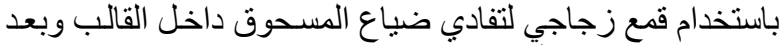
ذلك تسـليط ضـغط أحسادي الاتجـاه مقداره (700Mpa) و الإبقاء

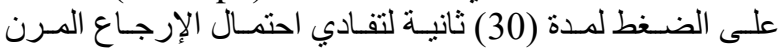

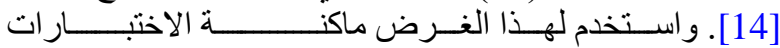




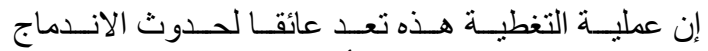

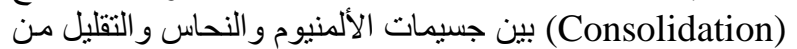

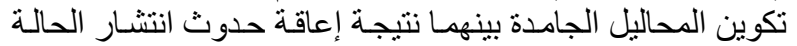

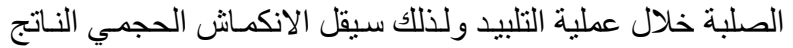

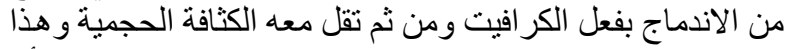

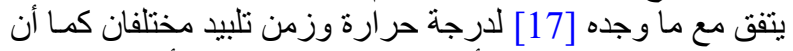

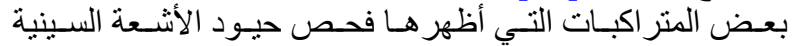

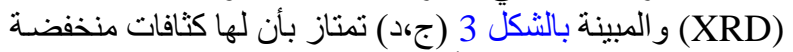

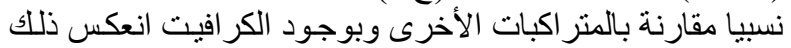
على خفض الكثافة الحجمية.

\section{الصلادة}

يبين شكل 5 العلاقة بين نسبة مادة التقوية والصلادة

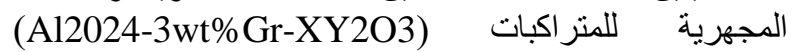

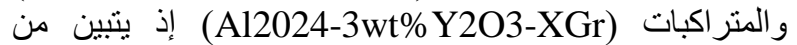
خلال الثكل أن زيادة محتوى جسميات التقوية (Y2O3)

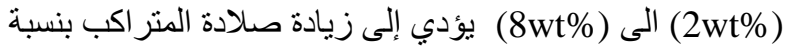

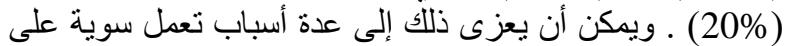

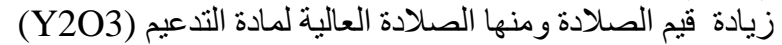

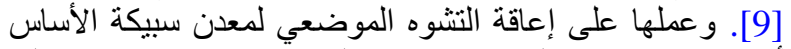

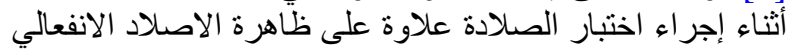

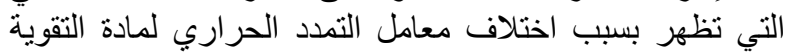

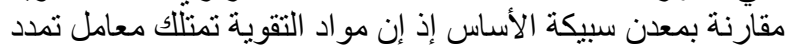

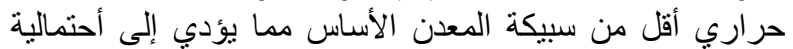

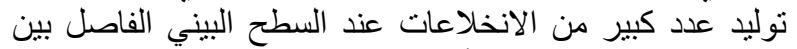

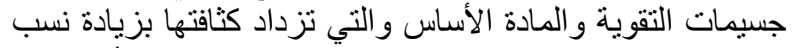

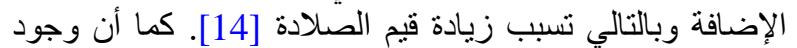
عنصر النحاس الذي يعمل على تكوين أطوار بينية شكل 3 (أكبات الإن) تزيد من الخواص الميكانيكية وهذا يتفق مع ما سبق التئ التوصل التهل إليه في دراسات سابقة من تأثنير مواد التقوية على الثى صلئ صلادة الألمنيوم وسبائكه [16] ولظروف تحضير مختلفة. أما بالنسبة للمنر اكبات فيلاحظ من شكل 5 (Al 2024-3wt\% Y2O3-XGr)

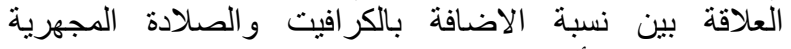

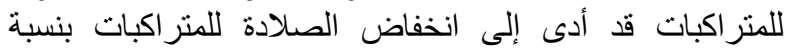

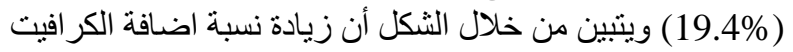

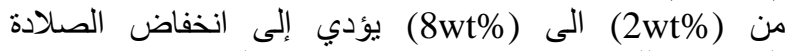

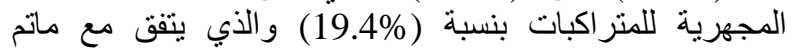

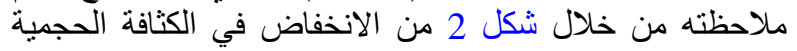

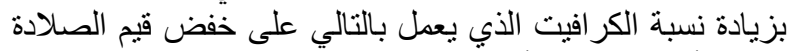

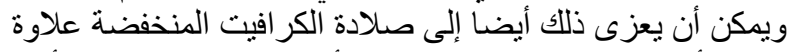

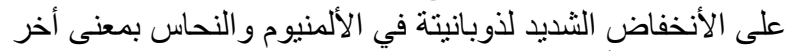
عدم حدوث أي اصلاد يذكر بالمحاليل الجامدة بين الالمنيوم والكرافيت من جها: Solution Hardening) و النحاس و الكر افيت من جها اخرى.

\section{مقاومة الانضغاط}

يبين شكل 6 العلاقة بين نسبة التقوية باليتيريا (Y)

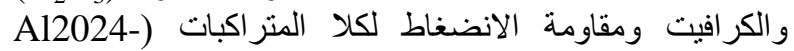

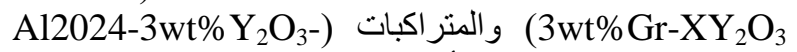
(XGr

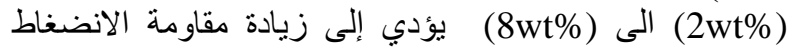

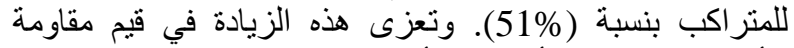

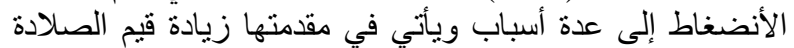

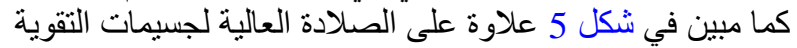
التي تعمل على توليد الانخلاعات و إعاقة حركتها

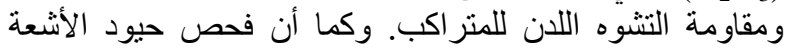
السينية شكل 3 (أ،ب) قد أظهر وجود بعض وكن الأطوار التي تتميز
باعتماد طريقة المسمار على القرص (Pin-on-Disk) أما الكثافة

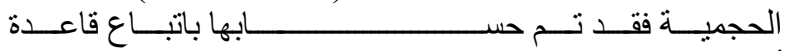

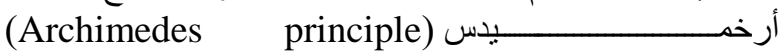

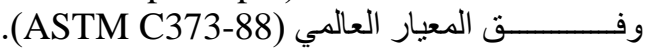

\section{4. النتائج والمناقشة الكثافة الحجمبية}

يبين شكل 2 العلاقة بين نسبة التقوية و الكثافة الحجمية

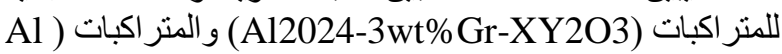
(2024-3wt\% Y2O3-XGr المساحيق إذ إن (Wt\%) (X=0,2,4,6,8) ويتبين من خلال الشكل

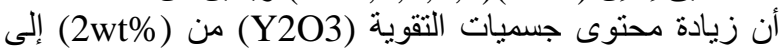

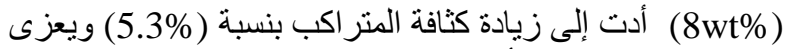

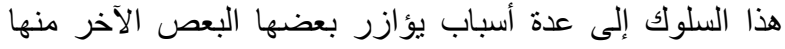

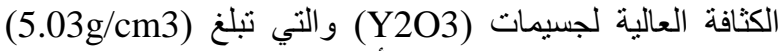
مقارنة مع كثافة معدن سبيكة الأساس (6l 2024-3wt\%Gr)

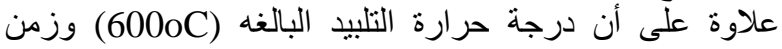

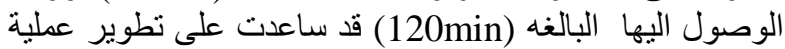

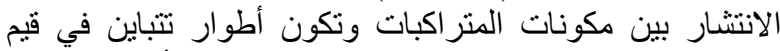

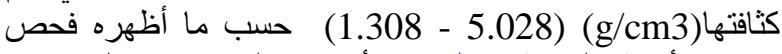

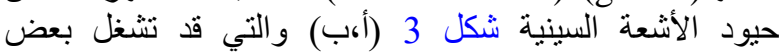

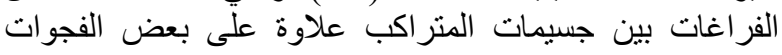

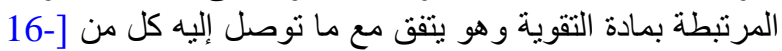

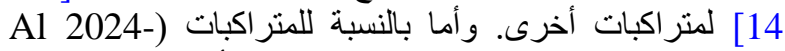
3wt\%Y2O3-xGr الكرافيت ادى إلى انخفاض الكثافة الحجمية للمنر اكبات بنسبة

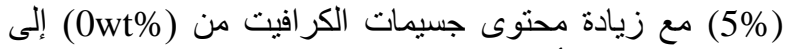

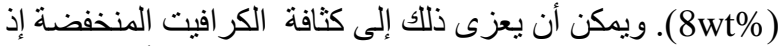

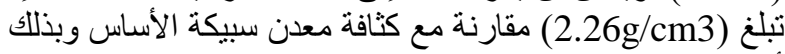

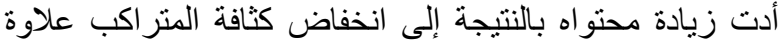
على دور الكرافيت الذي يتمثل في تكوين تجمعات (cluster)

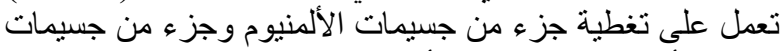

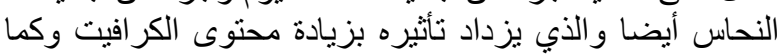
موضح في صور البنية المجهرية شكل 4.

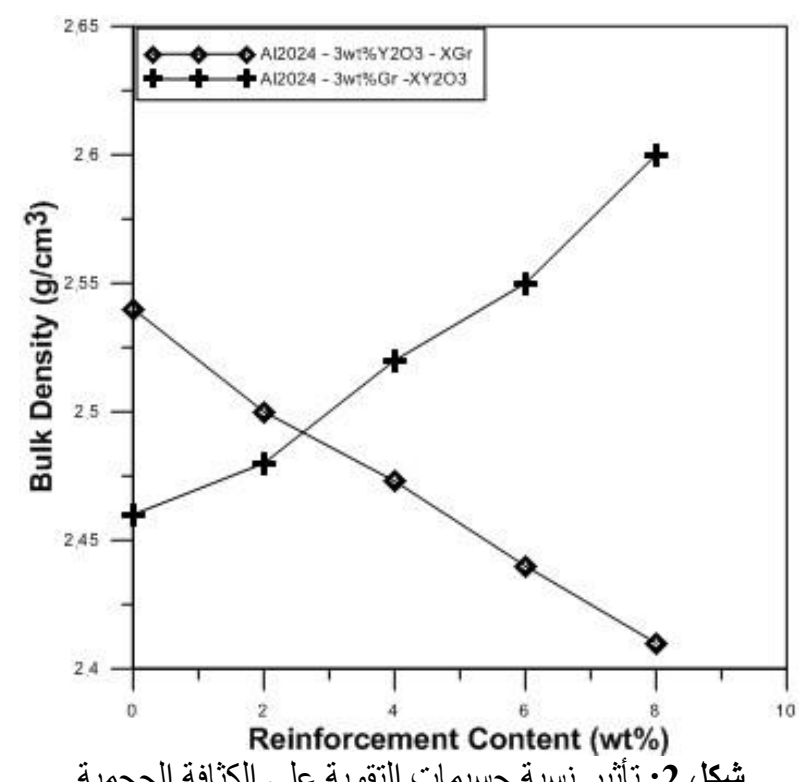

شكل 2: تأثير نسبة جسيمات التقوية على الكثافة الحجمية للمتر اكبات. 
الكر افيت ستعمل على تغليف و عزل بعض من جسيمات المتر اكب الأمر الذي يقلل من عملية الاندماج بينها وهو بئن يتفق مع ما أثنار

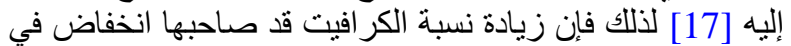

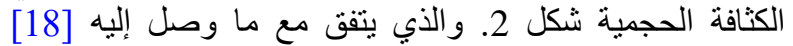

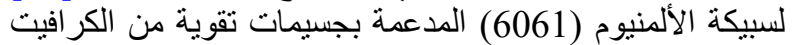

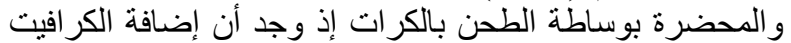
إلى المتر اكبات ذات الأساس المعدني يقلل من مقاومة الانضغاط.

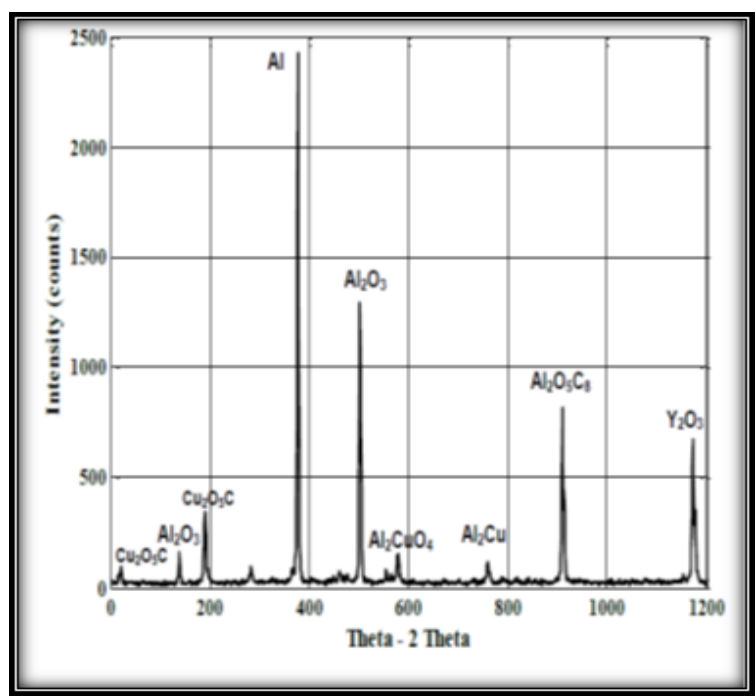

$(+)$

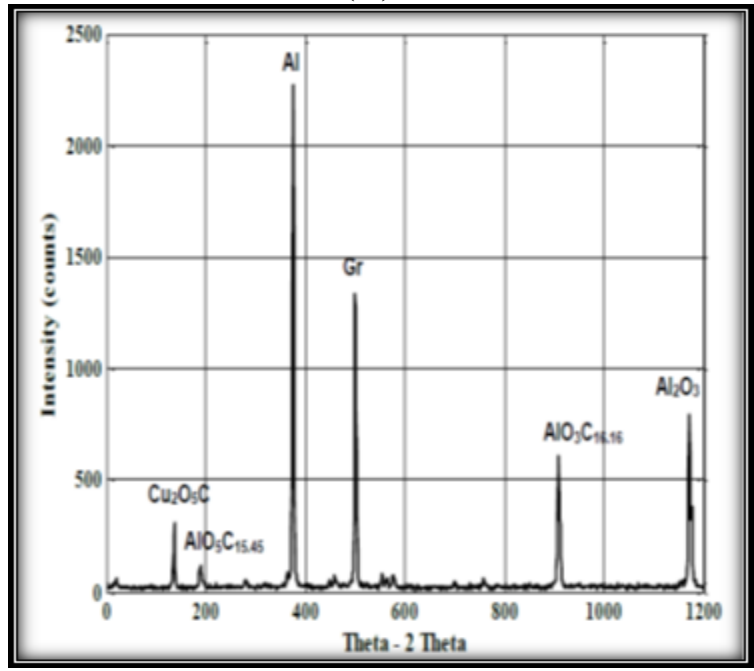

(د)
بكثافتها العالية التي تساهم في زيادة الكثافة الحجمية شكل 2 التي

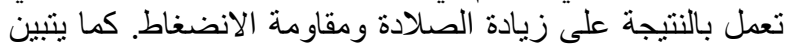

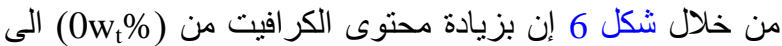

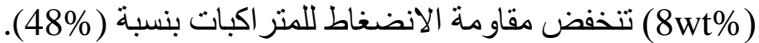

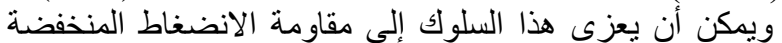

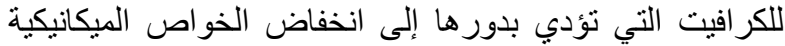

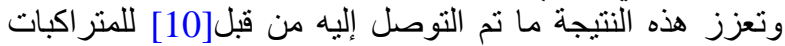
بنسب وظروف تحضير متباينة. علاوة على أن زيادة نسبة لمبن

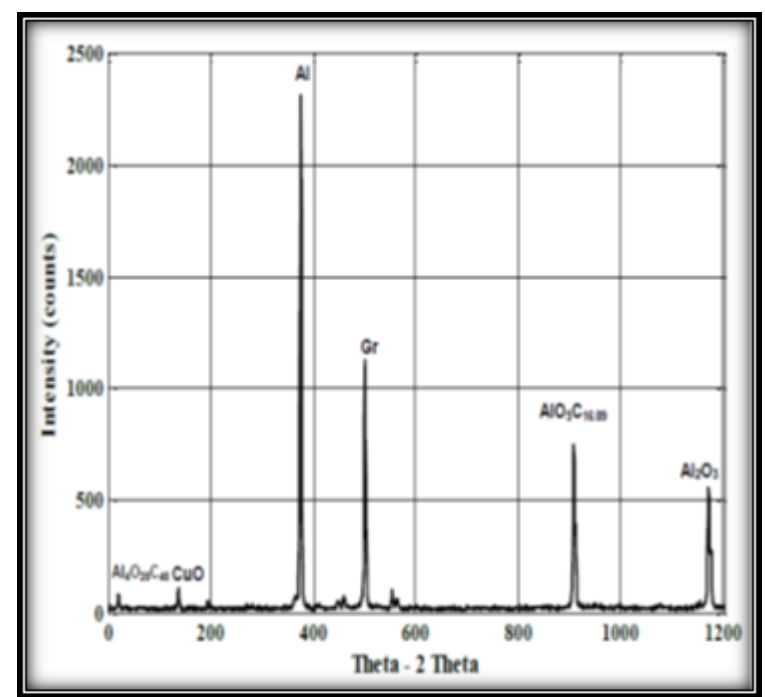

(i)

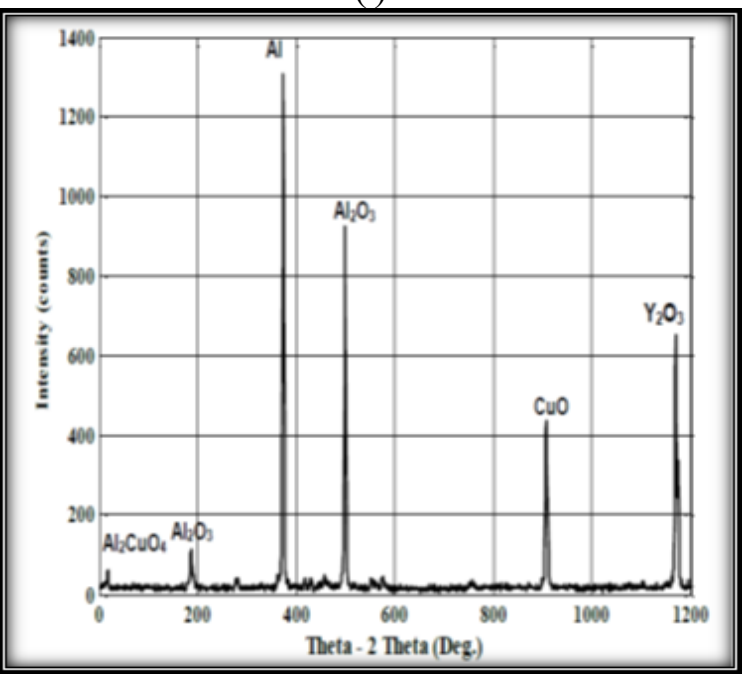

(ج)

شكل 3: فحص حيود الأشعة السينية لبعض المتر اكبات (أ) Al2024-3wt\%Gr-0wt\%

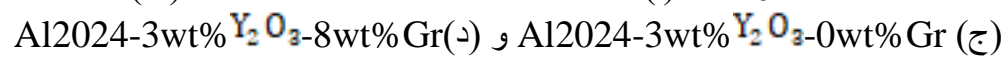

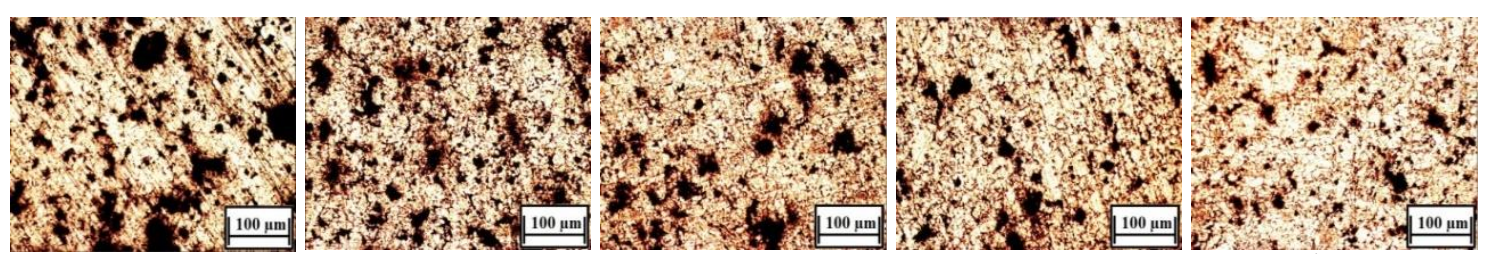

(j)
(ج)
(أ)

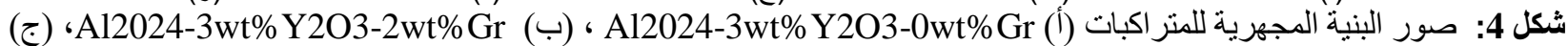
Al2024-3wt\% Y2O3-8wt\%Gr (j) و Al2024-3wt\%Y2O3-6wt\%Gr (د) ،Al2024-3wt\%Y2O3-4wt\%Gr 


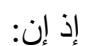

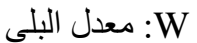

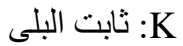

N N الحمل العمودي المسلط

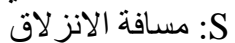

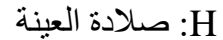

C عامل هندسي بعتمد على البنية المجهرية النية

ومن شكل 7 يلاحظ إن معدل البلى للمتر اكبات

(Al2024-3wt\% أقل من من معدل البلى البلى

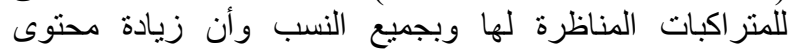

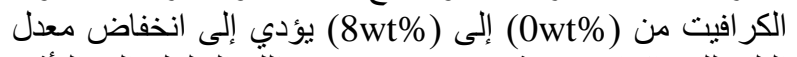

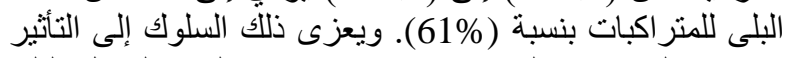

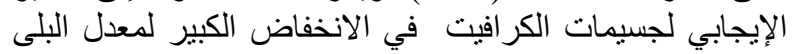

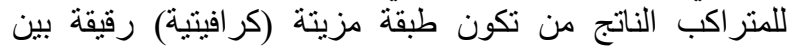

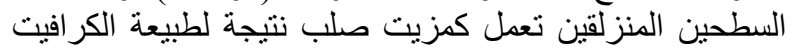

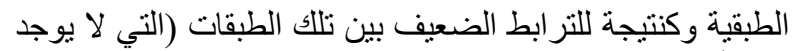

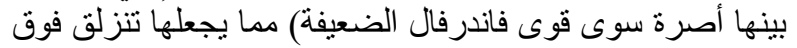

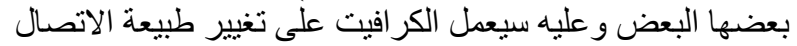

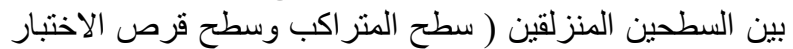

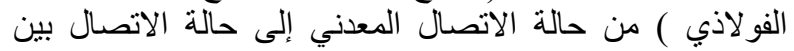

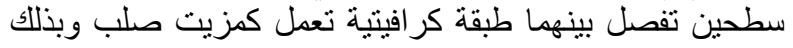

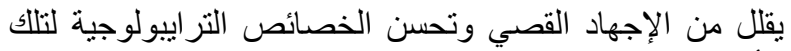

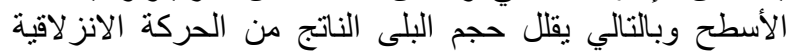

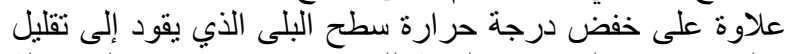

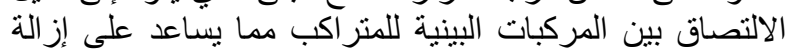

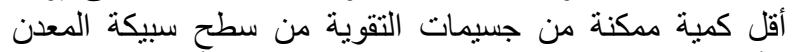

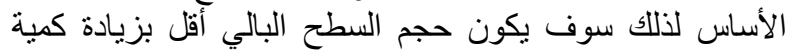
الكر افيت و هو ما توصل إليه [21] عند التقوية بكاربيد السليكون

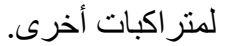

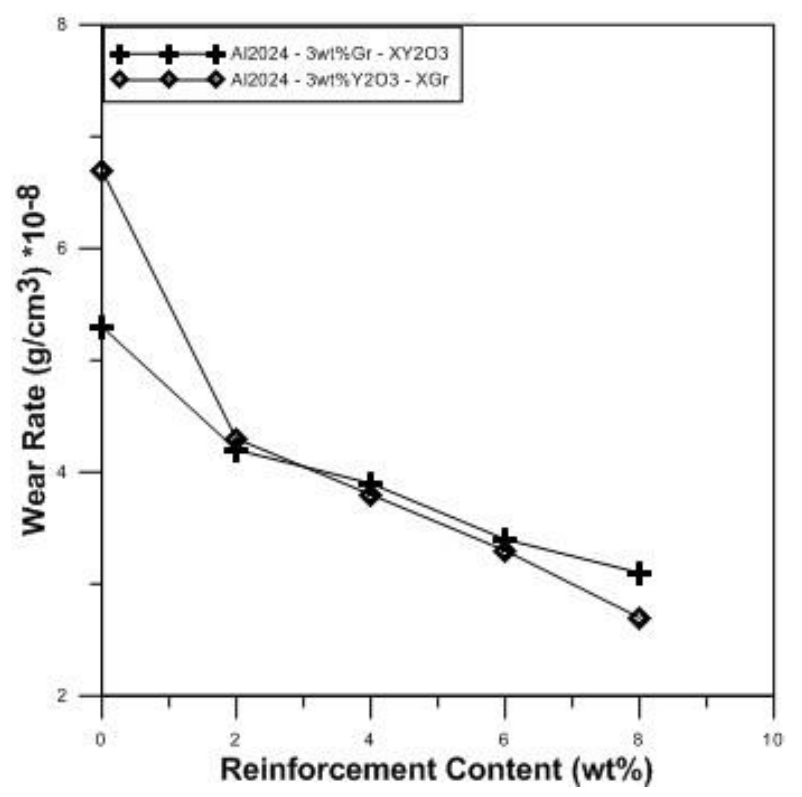

شكل 7: تأثير نسبة جسيمات التقوية على معدل البلى للمتر اكبات

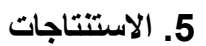

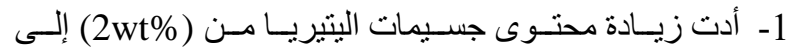

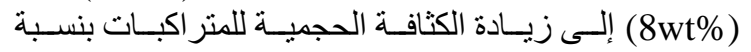

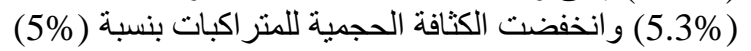
مع زيادة محتوى الكر افيت حتى الوصول إلى نسبة إضـافة

.(8wt\%)

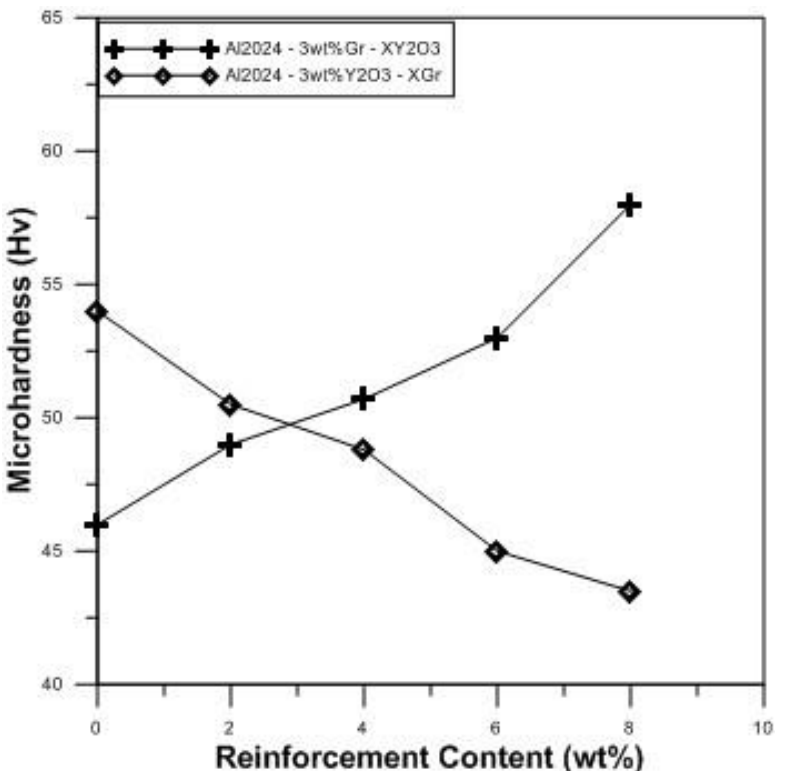

شكل 5: تأثير نسبة جسيمات التقوية على الصلادة المجهرية ل المتر اكبات

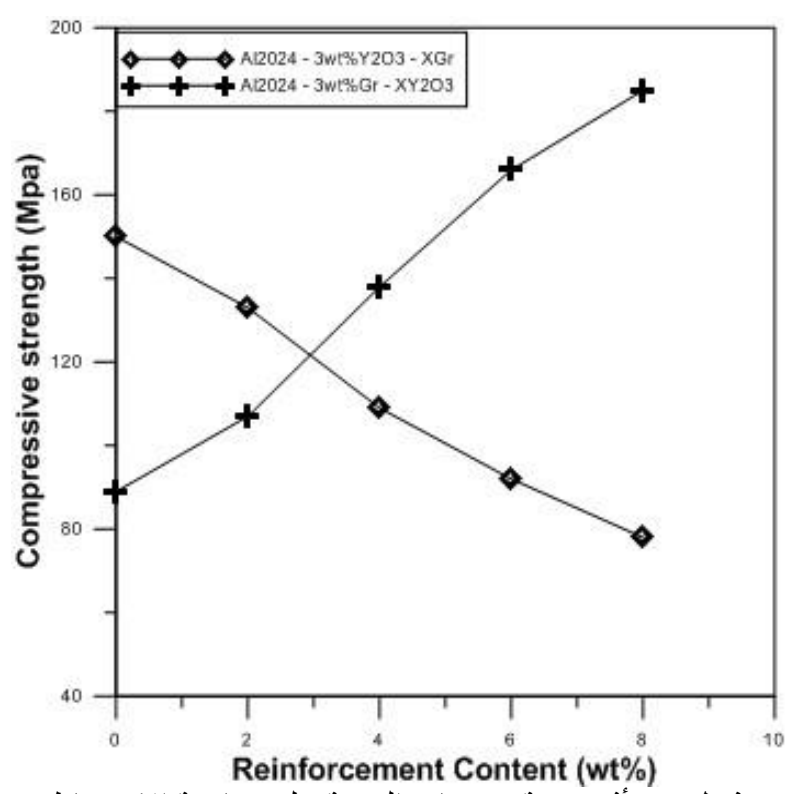

شكل 6: نأثير نسبة جسيمات التقوية على مقاومة الانضغاط

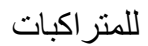

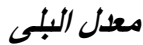

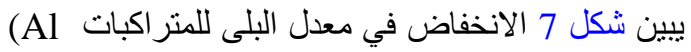
Al 2024-) 2024-3wt\% Gr-XY2O3) 3wt\% Y2O3-XGr

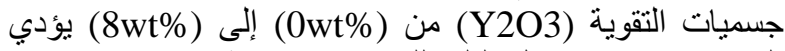

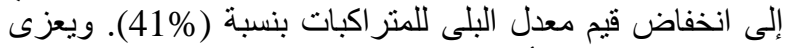

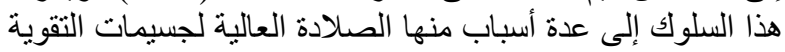

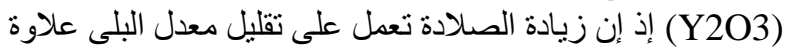

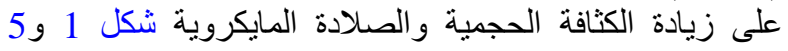
و هناك علاقة عكسية بين معدل البلى ومتوسط الصلادة المادية المايكروية

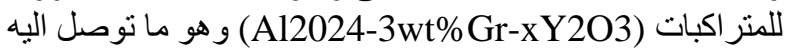
[19] عند التقوية بكاربيد السليكون والذي يتفق مع العلاقة

$W=K \frac{N S}{C H}$ 
Indian Institute of Metals 2010;63(5): 813817.

[12] Ravindran P, Manisekar K, Narayanasamy P, Selvakumar N, Narayanasamy R. Application of factorial techniques to study the wear of al hybrid composites with graphite addition. Materials and Design 2012;39:42-54.

[13] سيف صباح ارحيم .دراسة تاثير متغيرات ميتالورجيا المساحيق على الخو اص الديكانيكية والفيزياوية لمتر اكبات

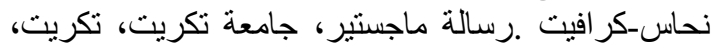
العر اق: 2013.

[14] Mahdi FM, Annas AA. Effect of yttria content on mechanical properties of $\mathrm{Al}$ Y2O3 composites prepared via squeeze casting and powder metallurgy routes. Sulaimani Journal for Engineering Science 2015;2(2):

[15] Kim SCH, Kim MT. Effect of copper addition on the sintering behavior and mechanical properties of powder processed AL/Sic composites. Journal of Materials Science 2005;40:441-447.

[16] Vinoth kumar SV, Manisekar K, Ravindran P. Development and tribological performance of nano Sic particles on The AA2024 hybrid composites with the addition of nano graphite. 5th International \& 26th conference 2014 December 12-14; All India Manufacturing Technology, India.

[17] Goytia-Reyes R， Gallegos-Orozco V. Microstructure and properties in AL-C-Cu system produced by mechanical milling. Journal of Alloys and Compounds 2009;485:837-842.

[18] Son HT, Kim TS. Homogeneous dispersion of graphite in a 6061 Aluminum alloy by ball milling. Materials Science and Engineering 2003;A348:163-169.

[19] Liu ZY, Wang QZ, Xiao BL. Experimental and modeling investigation on $\mathrm{SiCp}$ distribution in powder metallurgy processed $\mathrm{SiCp} / 2024 \mathrm{AL}$ composites. Materials Science and Engineering 2010 A527: 5582-5591.

[20] Moustafa SF, El-Badry SA. Friction and wear of copper-graphite composites made with $\mathrm{Cu}$-coated and uncoated graphite powders. Journal of Wear 2002;253: 699710.

[21] Ravindran P, Maniseker K. Tribological behavior of powder metallurgy processed aluminum hybrid composites with the addition of graphite soild lubricant. Ceramic International

2013;39:1169-1182.

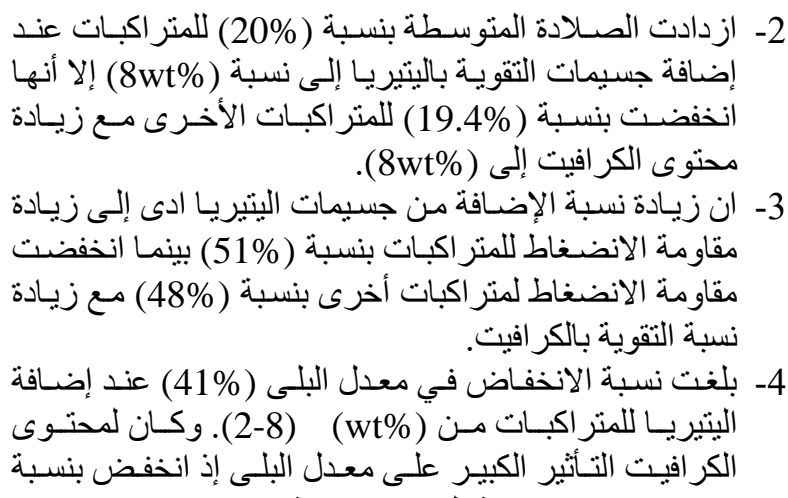

[1] Upadhyaya GS. Powder Metallurgy Technology, England, 2002.

[2] Bansala P, Upadhyayb L. Experimental investigations to study tool wear during turning of alumina reinforced aluminum composite. Procedia Engineering 2013;51:818-827.

[3] Moustafa SF, Abdel-Hamid Z. Copper matrix $\mathrm{Sic}$ and $\mathrm{Al} 2 \mathrm{O} 3$ particulate composites by powder metallurgy technique. Materials Letters 2002;53:244-249.

[4] Karl UK. Metal matrix composits. WileyVCH Verlag GmbH \& Co. KGaA, 2006.

[5] Chawla N, Chawla KK. Metal matrix composites, Springer, 2006.

[6] Harris B. Engineering composite materials. The Institute of Materials, London, 1999.

[7] محمد سعيد وحيد، سدير موفق مجيد. در اسة معدلات التأكل

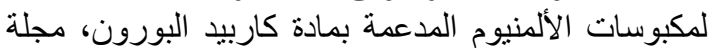

$$
\text { العلوم والتكنلوجيا } 2010 \text { 28 28(18): }
$$

[8] Bhargavi R, Ramanaiah N. Studies on mechanical properties of 2024Al-B4C composites. Advance Materials Manufacturing \& Characterization 2014;4(1):42-46.

[9] Bouaeshi WB, Li DY. Effect of Y2O3 addition on microstructure mechanical properties electrochemical behavior and resistance to corrosive wear of Aluminum. Tribology International 2007;40:188-199.

[10] Akhlaghi F, Zare-Bidaki A. Influence of graphite content on the dry sliding and oil impregnated sliding wear behavior of Al2024-graphite composites produced by insitu powder metallurgy method. Wear 2009;266:37-45.

[11] Yarra R, Venkatachalam P. Densification of $\mathrm{Al} / \mathrm{Y} 2 \mathrm{O} 3$ composite powder by equal channel angular pressing. Transaction of 
Raed N. Razooqi, Ahmed A. Akbar, Mohanad L. Hamada/ Tikrit Journal of Engineering Sciences (2019) 26(4) 1-7 